\title{
Intermediate Outcomes, Strategies, and Challenges of Eight Healthy Start Projects
}

\author{
Andrea Brand · Deborah Klein Walker • \\ Margaret Hargreaves $\cdot$ Margo Rosenbach
}

Published online: 15 November 2008

(C) Springer Science+Business Media, LLC 2008

\begin{abstract}
Site visits were conducted for the evaluation of the national Healthy Start program to gain an understanding of how projects design and implement five service components (outreach, case management, health education, depression screening and interconceptional care) and four system components (consortium, coordination/collaboration, local health system action plan and sustainability) as well as program staff's perceptions of these components' influence on intermediate outcomes. Interviews with project directors, case managers, local evaluators, clinicians, consortium members, outreach/lay workers and other stakeholders were conducted during 3-day in-depth site visits with eight Healthy Start grantees. Grantees reported that both services and systems components were related to self-reported service achievements (e.g. earlier entry into prenatal care) and systems achievements (e.g. consumer involvement). Outreach, case management, and health education were perceived as the service components that contributed most to their achievements while consortia was perceived as the most influential systems component in reaching their goals. Furthermore, cultural competence and community voice were overarching project components that addressed racial/ethnic disparities. Finally, there was great variability across sites regarding the challenges they faced, with poor service availability and limited funding the two most frequently reported. Service provision and systems development are both critical for successful Healthy Start projects to achieve intermediate program
\end{abstract}

A. Brand $(\varangle) \cdot$ D. K. Walker · M. Hargreaves

Abt Associates Inc., Cambridge, MA, USA

e-mail: andrea_brand@abtassoc.com

M. Rosenbach

Mathematica Policy Research, Inc., Cambridge, MA, USA outcomes. Unique contextual and community issues influence Healthy Start project design, implementation and reported accomplishments. All eight projects implement the required program components yet outreach, case management, and health education are cited most frequently for contributing to their perceived achievements.

Keywords Infant mortality - Health disparities .

Consumer voice Cultural competence .

Systems development - Case management - Outreach . Health education · Consortium

\section{Introduction}

Infant mortality is a key indicator often used to compare health systems and health status worldwide. The U.S. infant mortality rate in 2004 was 6.78 infant deaths (under 1 year of age) per 1,000 live births, ranking below many developed countries [1]. However, within the United States, the infant mortality rates vary greatly by race/ethnicity, painting an even grimmer reality. Among infants born to non-Hispanic black mothers, the infant mortality rate in 2004 was 13.60 , compared to the infant mortality rate of 5.66 for infants born to white mothers, with the mortality rates for infants born of mothers who are American Indian and Puerto Rican falling between the two [1].

In response to the major racial disparities in infant mortality, the national Healthy Start program began in 1991 as a 15 -site demonstration project and evolved over the past 16 years into a major maternal and child health community program in 37 States, the District of Columbia, and Puerto Rico. Currently in its fourth funding cycle with 96 projects and total funding approximately $\$ 100$ million 
per year, Healthy Start is designed to eliminate disparities in infant mortality and other birth outcomes through the implementation of nine required program components within the context of the community. The five service components (outreach, case management, health education, perinatal depression screening, and interconceptional care) are intended to help improve access to care and birth outcomes by increasing women's knowledge and practice of healthy behaviors and available resources, and by ensuring the coordination of care for women and infants. The systems components (consortium, local health system action plan, collaboration with Title V, and sustainability planning) are intended to help mobilize the community to improve perinatal health by ensuring that social and medical services are available to support pregnant and interconceptional women and their infants.

In the first phase of the current national evaluation of this cycle of 96 programs, a comprehensive survey of all 96 project directors resulted in a descriptive portrait of the entire national Healthy Start program [2]. In the second phase of the national evaluation of Healthy Start, intensive case studies were developed for a subset of eight grantees to gain a greater understanding of how they design and implement their individual projects and their reported intermediate program outcomes. This evaluation relies on a logic model developed to illustrate how the required program components are hypothesized to lead to the achievement of intermediate outcomes and ultimately, longer term improved maternal and child health outcomes (Fig. 1). The logic model was developed for the national evaluation in collaboration with key stakeholders in the Healthy Start program, including grantees, members of the Healthy Start Evaluation Panel and the Secretary's Advisory Committee on Infant Mortality.

Three program goals of Healthy Start are: (a) to reduce racial and ethnic disparities in access to and utilization of health services, (b) to improve local health care systems, and (c) to increase consumer/community voice and participation in health care decisions [3]. Achievement of these program goals are expected to result in population changes, or specifically: (a) improved birth outcomes, including infant mortality, birthweight, gestational age, and prenatal care adequacy; (b) improved maternal health, including health status and health risk behaviors; (c) increased time period between pregnancies or births; and (d) improved child health, including use of recommended health care services, immunization status, and health status. What the logic model does not capture is the continuously changing social and political climate in which Healthy Start grantees operate such as growing rates of poverty, State Medicaid eligibility criteria, immigration laws, demographic shifts in grantee target populations, and the lack of available services. These pose challenges to grantees' ability to implement the required Healthy Start program components.

This paper is one component of the recent national Healthy Start program evaluation, which used both qualitative and quantitative methods to assess program implementation, outcomes, and lessons learned. It presents the qualitative findings from the site visits with eight Healthy Start projects. A companion paper presents the results of a participant survey of women served by these eight projects.

\section{Methods}

We conducted site visits to eight selected Healthy Start projects, the purpose of which was to gain a full understanding of how specific Healthy Start program elements are implemented to achieve program goals and ultimately improve perinatal outcomes and reduce disparities. Specifically, our aim was: (a) to learn how different Healthy Start features are designed and implemented, factors associated with successful implementation, and the effects the program features have on service and systems outcomes; (b) to determine how services are coordinated within Healthy Start and with community providers; (c) to understand grantee perceptions of their achievements; and (d) gain stakeholders' perspectives on the barriers to achieving goals at the individual service and system levels.

\section{Selection of Eight Grantees}

The eight grantees included in the site visits were selected based on primary and secondary analyses of data obtained from 96 grantees funded in 2001, including responses to the 2004 National Survey of Healthy Start Programs collected in the first phase of the evaluation and performance measurement data reported to HRSA's Maternal and Child Health Bureau. Criteria to be considered for the case study sample included: (a) completion of the 2004 National Survey of Healthy Start Programs, (95 grantees), (b) implementation of all nine required components of the Healthy Start program (55 grantees), (c) capability to track referrals to providers within and outside of Healthy Start (39 grantees), and (d) maintenance of electronic records (27 grantees). In addition, the sample was selected to ensure that there was representation from each of the four U.S. census regions as well as representation that included a rural project, a project located near the United States/Mexico border, and a project that served a predominantly indigenous (American Indian) population. The dataset was then sorted by funding level, total live births gathered from reported performance measure data, census region, and catchment area.

Of the 27 eligible grantees, a purposive sample of eight grantees and eight alternates was selected using a non- 


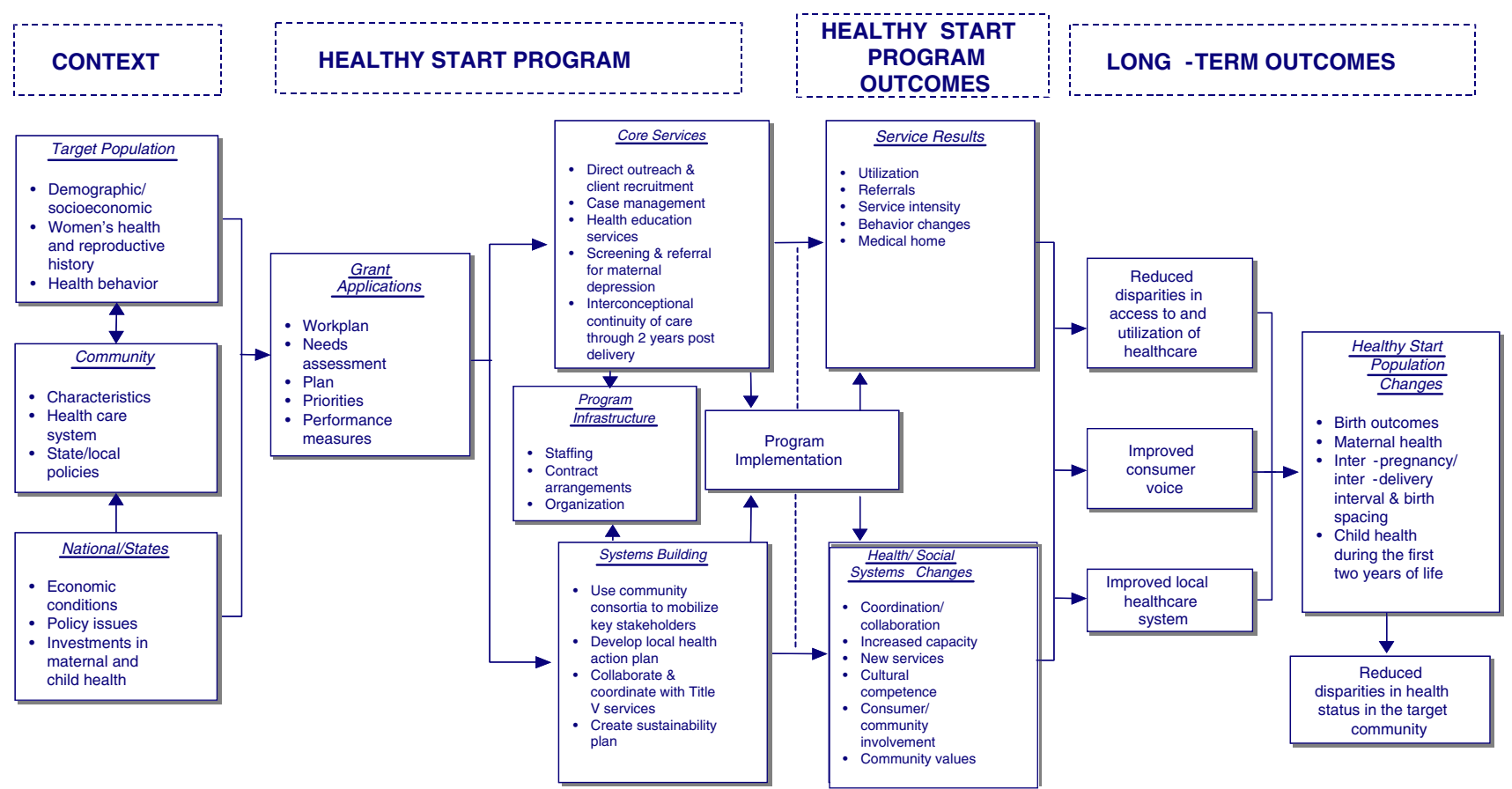

Fig. 1 Healthy start logic model

probability sampling scheme [4]. This approach provides a sample that can be analyzed to obtain results and make inferences about the selected sample only. This sample cannot be used to generalize results to the larger set of 96 grantees since it is not representative of the larger group of grantees. The grantees selected include projects located in Fresno, CA; Tallahassee, FL; Des Moines, IA, E. Baton Rouge, LA; Worcester, MA; Las Cruces, NM; Pittsburgh, PA; and Lac du Flambeau, WI.

\section{Instrument Development}

Using the findings from the 2004 National Survey of Healthy Start Programs [2] as a launching point, discussion guides were developed to elicit feedback from multiple stakeholders at each project. To reach a broad array of key stakeholders, separate discussion guides were created for the project directors, case managers/care coordinators, outreach workers, clinicians (e.g., physicians, social workers, and other mental health providers), local evaluator/MIS manager, consortium members (including consumers), and other stakeholders (e.g. regional public health officials, State Title V directors).

\section{Data Collection}

Three-day site visits in 2-person teams included over $21 \mathrm{~h}$ of interviews per site. In addition to discussions with key stakeholders, two additional activities were included during each site visit. First, a participant flow exercise was conducted with a group of case managers and outreach workers. The purpose of this exercise was to gather systematic data on how participants flow through the service system from the point of entry. Second, a relational mapping/prioritization exercise was conducted with the project director to obtain her understanding of the relationship between program components and their contribution to the project's achievements. Each project director created a visual diagram of the project structure depicting the nine core components of the Healthy Start program as well as additional features that were important to their project. A series of questions about the diagram provided detailed information about the project. All of the information collected during the on-site interviews was synthesized into eight separate site summary reports.

\section{Data Analysis}

Using standard techniques for qualitative analysis [5], cross-site analyses were conducted with the Healthy Start logic model serving as an organizing framework. We began by organizing information from the eight site visits into 11 major content areas, comprised of the nine required program components as well as two components that were relevant to community programs: cultural competence and community involvement/voice.

We took verbatim responses from each of the interview respondents at the eight sites. Responses were grouped 
according to topic area or module, coded, and cross-coded if they overlapped categories. Categories for coding were developed before data analysis and additional codes were created during the analysis. Reliability checks were conducted through blinded recoding of responses. This led to the creation of the master files that were used to organize and analyze the qualitative data collected across sites. The resulting qualitative dataset provided a structural base from which the findings emerged.

We examined the information across sites, disaggregated by subtopics used in the site visit discussion guides. We then compared responses across projects to determine differences across sites regarding how they implemented the 11 major content areas and created theme tables to note any patterns that emerged. Data from the mapping exercise were organized into a data table, by site, to examine how project directors gauged the level of importance of the various program components. The mapping tables from all of the sites were combined into a single aggregate table to create a picture of which components project directors attribute with success. Next, we examined the self-reported accomplishments reported by the projects. Accomplishments need only be reported by a single respondent at a project site in order to be captured. These accomplishments were then coded to identify common themes and were then summarized in a table. We used the same technique for analyzing the sites' reported challenges.

The logic model (Fig. 1) illustrates our hypothesized model of change for how Healthy Start program activities lead to reduced disparities in birth outcomes. The nine basic required components of services and systems will produce Healthy Start individual results such as utilization of services and behavior changes as well as health systems changes resulting in community voice and cultural competence. Eventually, these intermediate outcomes will produce long-term outcomes such as reduced disparities in utilization of services, improved consumer voice, improved health care systems and, finally, improved birth outcomes and reduction of health status disparities.

Our findings provide an in-depth snapshot of how the eight Healthy Start programs implement the nine program components to meet the needs of the most vulnerable women and their families in their communities. We start with an examination of the each component, describing the diversity and similarities of how they are implemented as well as the barriers encountered. We also include a description of how programs achieve cultural competence and community involvement/voice, two themes which emerged from our cross-site analysis as critical to the successful implementation of Healthy Start and reported program outcomes. Cultural competence has received growing attention as a feature in eliminating racial/ethnic disparities in health care [6]. Community involvement, though not a core requirement of the funded projects, is an area that has been recognized by the national Healthy Start program as influencing change [7]. Community involvement refers to the participation of Healthy Start service consumers on local consortia, and employing community residents as paraprofessionals.

\section{Results}

\section{Service Components}

\section{Outreach}

Our analysis reinforced the hypothesis or finding that outreach is one of the cornerstones of Healthy Start projects. Outreach is the provision of case finding services that actively recruit and retain participants from a project area into a comprehensive system of care [3]. Although outreach efforts are uniquely provided by each of the projects in terms of the target population, recruitment tactics, and retention strategies, there are similarities that cut across all of the projects. For example, all projects use paraprofessionals or lay community workers to conduct outreach (although two additionally use formally trained professionals such as nurses and social workers) and employ multiple strategies to reach potential participants. Strategies include visits to community settings such as churches, hospitals, and health fairs to distribute outreach materials or make presentations. As one respondent noted, "It is very effective to sit in front desks of hospitals, WIC, and clinics." Outreach targeted different populations; some projects targeted all women within a catchment area (four projects), while others focused on African Americans or other subpopulation with a history of poor birth outcomes (four projects). ${ }^{1}$

All the grantees use outreach efforts to retain enrolled participants. For most projects (six), these efforts included incentive-based practices such as the distribution of items or accumulation of points for participants to "purchase" tangible goods such as clothes, diapers, toothbrushes, and baby thermometers available in projects' storerooms. Half of the projects offered transportation to retain participants, and three projects offered childcare so women could stay involved.

Few challenges to outreach were noted by respondents. These included the inability of reaching moms with daytime jobs when the majority of outreach activity is conducted (two projects); undocumented participants who

\footnotetext{
${ }^{1}$ In this section, the number of projects with a particular response is mentioned in parentheses. Since responses are not always mutually exclusive, the numbers do not necessarily add up to eight.
} 
fear deportation (two projects); language barriers (two projects); and budget constraints which prohibit the provision of tangible goods to retain program participants (one project).

\section{Case Management}

Case management is the main link between participants and needed supports and services. Case management is provided to coordinate services from multiple providers to meet the needs of each participant through assessment, referral, monitoring, facilitation, and follow-up [2]. Case management is fundamental to Healthy Start projects; as one respondent stated, "Case management is the life thread of our project." All projects used a multidisciplinary case management approach with some mix of social workers, nurses, and paraprofessionals. Higher risk participants are assigned to nurses (three projects), have more frequent home visits (three projects), or both (one project). All projects include an initial risk assessment within their case management services, but the schedule for repeat assessments varies greatly, ranging in frequency from every encounter (two projects), every trimester (one project), twice a year (one project), annually (one project), to none (three projects). A combination of formal screening tools and informal assessments via observation are used.

Service planning is a major component of case management and involves some form of a written service plan to capture participants' goals (seven projects). This process typically involves participants (six projects), family members (four projects), and less frequently, other agencies (three projects). In the words of one respondent, "Allowing participants to be involved is the strength and why [service planning] works." Another respondent noted, "It is the tool that drives case management."

Projects attempt to provide case management during the interconceptional period. Whereas women receive case management anywhere from 2 months to 2 years postpartum, all projects indicated that case management for babies continues at least 2 years. The emphasis for babies is on infant health and nutrition, safety, and parent bonding; the emphasis for women is on spacing births at least 2 years apart (six projects). A new and emerging service in many programs focuses on male participation. Although formal male partner case management programs were not common, the importance of male involvement was encouraged informally by all projects (e.g. programs include them during home visits).

Challenges to case management include access barriers such as lack of transportation or childcare (five projects); a mobile or hard to reach population (four projects); large caseloads (three projects); limited access to mental health and substance abuse services (three projects); lack of resources to meet participants' basic needs (three projects); and participant distrust (two projects).

\section{Health Education}

Health education serves as a critical component of Healthy Start often within outreach and case management responsibilities. Health education consists of instructional activities designed to increase knowledge and awareness to influence behavior [3]. It is commonly offered by a team of individuals, thereby maximizing the opportunities to reach participants. Case managers in one project noted that their job is "all about education." Only two projects have staffed or contracted positions specifically charged with providing health education to participants and their families.

All projects indicated they provide health education through one-on-one contact, primarily in participants' homes, but also in the prenatal clinics. Group classes, including topics that focus on parenting and childbirth, are also used (five projects). Less common were community locations such as schools and faith-based organizations (three projects). In addition to delivering health information verbally and through written handouts, many projects indicated they use visual aids such as videos and picture handouts to promote education (six projects). Additionally, Healthy Start projects use opportunities such as outreach and transportation to impart health education messages (three projects). For example, one respondent noted that driving a woman to an appointment provided an ideal environment to offer health information.

The number of health education topics covered during the prenatal period ranged from as few as six to more than 20. Projects placed an emphasis on domestic violence (eight projects), stress management, preterm labor, family planning, HIV, smoking cessation, drug and alcohol abuse, nutrition (seven projects), STDs (six projects), breastfeeding (six projects), and parenting education (five projects).

The health education topics offered prenatally and during the interconceptional period did not vary greatly. Not surprisingly, however, the importance of birth spacing is emphasized more during the interconceptional period. Other topics commonly offered during the interconceptional period include parenting (seven projects), breastfeeding (five projects), and immunization (three projects). The success of health education was not systematically assessed by grantees, but was perceived to be effective.

Several challenges to providing health education were noted by respondents. The most common challenges were overcoming participants' resistance to accept information, their unwillingness to change, and their general disinterest in talking with a health educator (five projects). Other challenges included a lack of privacy to discuss sensitive 
topics (two projects), fear of outsiders, particularly among the immigrant population (one project); and inability to reach participants, especially in rural areas (two projects).

\section{Perinatal Depression Screening}

The purpose of the required perinatal depression screening component is to promote systems of care that address gaps in screening and assessment for depression during and around the time of pregnancy and to enhance linkages to community based intervention services for depression [3]. Healthy Start is the first national program to mandate depression screening. All eight projects indicated that case managers are responsible for depression screening. For those projects that use a professional/paraprofessional staff mix, depression screening falls to case managers with clinical degrees (nurses/ social workers). Most projects reported using a standardized instrument such as the Edinburgh Perinatal Depression Screening Tool (six projects) or the Center for Epidemiological Studies Depression Scale (one project). One project does not have a formal tool but integrates a battery of questions into their comprehensive risk assessment.

All projects screen all of their participants, although the schedule varies from only during the postpartum period (one project), to initial prenatal assessment and postpartum (three projects), to several screenings during and after pregnancy (four projects). However, even for those projects with less rigorous screening schedules, all projects indicated they screen "off-schedule" if a participant displays depression symptoms.

Several projects have made efforts to adapt their screening practices to meet the cultural needs of participants. These efforts include the translation of instruments into other languages (three projects), reading the tool to participants if necessary (one project), and rewording questions or defining terms to eliminate misunderstandings (one project).

For positive screens, all projects refer participants to mental health providers within the community. Respondents indicated they also notify a medical doctor (two projects), and intensify home visits (two projects) including one project which started offering Listening Visits, ${ }^{2}$ an alternative to counseling that feels more socially acceptable for some women. It involves case managers providing support by listening to and offering problem solving assistance. While not considered a form of therapy, Listening Visits are considered therapeutic.

Although depression screening has been embraced by all projects, the shortage of culturally competent mental health

\footnotetext{
${ }^{2}$ Listening Visits are modeled after the British Counseling Intervention Model used in the United Kingdom. Research in the UK indicates that these visits are very effective in improving women's overall mood and reducing the level of depression [8].
}

service providers remains a challenge, particularly for participants without health insurance (six projects). The lack of mental health services in languages other than English also interferes with participants' access (three projects). Other barriers include stigma (four projects) and transportation (two projects). To alleviate some of the demand, in-house counselors are available to a limited extent (two projects), but the need for community providers remains essential.

\section{Interconceptional Care}

Healthy Start is the first national program to emphasize the interconceptional period, defined as the time between the end of one pregnancy and the beginning of the next, or 24 months postpartum, whichever comes first [3]. Efforts to recruit participants into Healthy Start services during the interconceptional period typically do not focus on serving new participants but concentrate on keeping prenatal participants enrolled after they give birth (six projects). As one respondent stated, "We are always working on birth spacing and have a family planning component which is introduced at the first visit. This is how we recruit [for interconceptional care]." Two projects indicated that outreach to and referrals from various community agencies help enroll new participants during the interconceptional period.

Interconceptional care differs from prenatal care in two key ways: (1) case management/home visits tend to be less frequent; and (2) health education topics include infant care, immunization, and development, as well as greater emphasis on birth spacing of at least 2 years. Like the prenatal period, services for women during this phase include risk assessments for depression, domestic violence and substance abuse, but also emphasize contraceptive assistance. Additionally, three projects focus on establishing a medical home for the infants of women they serve.

Retaining participants during the interconceptional period is challenging, and projects rely on various practices to keep them engaged. Home visits, incentives such as small tangible items like calendars and thermometers, and health education are largely used to keep women involved. One project noted that retention is not a problem because participants don't want to leave. Participants do not want to forfeit the relationships they built with their case managers.

System Components

\section{Consortia}

A community-based consortium is a formally organized partnership, advisory board or coalition of organizations and individuals which identify themselves with the 
proposed target project area, and who collectively apply their resources to the implementation of a common strategy for the achievement of a common goal within that project area [3]. Healthy Start consortia play an important role by creating a venue for community members, participants, social service agencies, housing representatives, religious and activist groups, law enforcement, and healthcare providers to work with program staff to implement Healthy Start. Through consortia, Healthy Start programs gain community trust and credibility, while working together to improve the local service system. Four of the projects focus on strategic planning, public relations, inter-agency collaboration, service enhancements and cultural sensitivity within Healthy Start while three focus on health policy and larger systems change, and one concentrates on informing policy makers of consumer needs. Projects routinely devote efforts to training consortium members on a host of topics including infant mortality, birth spacing, domestic violence, postpartum depression, breastfeeding, smoking cessation, drug use, culturally competent healthcare, diabetes prevention, and tobacco cessation.

The eight grantees structure and organize their consortia differently to address their unique project needs. Six projects use a multi-pronged structure that includes at a minimum, two distinct entities-one with representatives of multiple agencies and organizations; and another comprised of consumers and other community members who are informally coached to learn how to participate in the agency-focused consortium. Another project uses a single advisory group that meets several times per year. This group is chaired by the project director, with planning assistance from staff members. Finally, one project, which is administered through the local health department, does not have a separate advisory body for Healthy Start. The mechanism for strategic planning, enhancing collaboration, increasing public awareness, and improving infant health is through two coalitions formed on specific topics-breastfeeding and substance abuse.

Project participants serve important roles on consortia in all eight projects, providing feedback on their experiences with the project, identifying challenges and benefits of the project, and serving as program advocates. As one project director stated, "It's important to have representation from the groups we're targeting, to make sure we have stakeholders from different venues." Similarly, a consumer involved in the consortium noted, "they ask me my opinion, and when I give it, they listen to me." To encourage consumer participation in consortia activities, projects have offered childcare, transportation assistance, meals, evening meetings, and language interpretation.

Despite concerted efforts, respondents reported that consumer involvement is thin (five projects). Barriers to participation include difficulty committing to regular attendance; access issues such as transportation and childcare; remoteness of the meeting location; and language barriers (one project respectively).

\section{Coordination/Collaboration with Title V and Other Entities}

Historically, an important element of Healthy Start's success is collaboration-is to promote cooperation, integration, and dissemination of information with Statewide systems and with other community services funded under the Maternal and Child Health Block Grant [3]. "Having a common goal, common direction for a common outcome makes a successful collaboration," stated one respondent. Through close coordination and collaboration with myriad service and system partners, the eight Healthy Start projects offer a wide range of services to address the needs of the target population.

All eight Healthy Start projects collaborate with State Title V programs through various mechanisms. Most collaboration takes place primarily through involvement of Title V agency staff on the Healthy Start consortia and participating in joint training activities for stakeholders with Healthy Start, including cultural competence training. Other frequently noted collaborative activities with Title V include having memoranda of understanding that formalize their involvement, developing consistent health messages for participants, using shared protocols for intake or risk/ needs assessment, participating in sustainability planning, and having a mutual data-sharing arrangement.

Healthy Start projects also collaborate with many other local health-related organizations, service-related providers, and community or civic-related entities using both formal and informal mechanisms. Respondents, in particular, noted their collaborative relationships with obstetricians, pediatricians, and nurse midwives, as well as hospitals, community health centers, and specialty clinics, WIC, Medicaid and SCHIP programs, mental health agencies and substance abuse treatment services. As one respondent noted, "The more collaborations you have, the more buy-in you have." Among the formal methods used, the Healthy Start consortium is most commonly used to bring different agencies and organizations together. Projects also rely on informal communiqués such as phone calls and referrals between agencies to foster relationships. Few challenges were reported but include poor communication between collaborative partners (two projects) and different approaches to systems change (one project).

\section{Local Health System Action Plan}

The 2001 HRSA Healthy Start Guidance defines the local health system action plan (LHSAP) as a four-year plan that describes ongoing collaborative mechanisms and planned 
efforts to work with existing community services to make improvements toward an integrated system for perinatal care for the target population [3]. The LHSAP is designed as a planning vehicle that links the strategic plans of Healthy Start programs to larger, geographically based public health planning efforts. The LHSAP includes goals within maternal and child health and objectives and strategies to reduce disparities in infant mortality through the coordination of Healthy Start services and local partners' activities.

All eight Healthy Start projects participated in some form of LHSAP planning, though efforts typically originated at another entity including the local public health authority (three projects), the consortium (two projects), a local task force to reduce infant mortality (one project), a non-profit organization charged with creating universal access to care (one project), and the Indian Health Services (one project). In seven of the projects, Healthy Start managers and staff assisted with the development of the LHSAP. These projects reported two kinds of system benefits from their involvement: (1) vertical linkage between Healthy Start activities to larger county and state maternal and child health efforts; and (2) collaborative action across agencies at the local level on specific issues targeted by the plans. Challenges for this component include unawareness by key informants that a LHSAP exists (one project); and the slow progress in completing a LHSAP (one project).

\section{Sustainability Planning}

A sustainability plan is defined as a set of administrative actions designed to identify and negotiate the continued financing and/or transition of project components to other entities to continue the provision of services in the project area beyond the Federal Healthy Start funded project period [3]. Healthy Start grantees spent less time on sustainability planning than any of the other service and system components. All eight of the Healthy Start projects have received reduced federal Healthy Start funding due to Congressional budget reductions each year. A combination of strategies is used to sustain services including replacing lost federal funding with other sources, transferring current services to other local providers, and increasing the efficiency of the current service system by reducing duplication. In particular, two projects are working with local coalitions to protect, enhance, or leverage state appropriations for Healthy Start or maternal and child health $(\mathrm{MCH})$ services. Three projects are writing grant applications for supplemental funding from state and foundation sources. Three projects established additional funding streams through entitlement programs such as billing Medicaid for case management services provided by a nurse and using Early Periodic Screening, Diagnosis, and Treatment (EPSDT) dollars to assist with transportation costs. Some projects are working with local partners to create system efficiencies by using economies of scale to provide more health care for women and infants (one project), finding ways to prevent service duplication within the community (one project), institutionalizing Healthy Start practices in the provider community such as the psychosocial risk assessment (two projects), and transferring efforts initiated by local Healthy Start to the community for ongoing funding and implementation, such as teen pregnancy prevention (one project).

Healthy Start consortia vary in their level of participation and leadership in sustainability planning. While some consortia have organized sustainability committees or have helped to identify potential grants and philanthropic opportunities (five projects), other consortia have continued to focus exclusively on service planning (two projects). Challenges to sustainability are described by respondents having noted which components would be effected by federal cuts in funding. Three projects noted having to reduce services in case management, health education, outreach, and depression screening. One project cited the elimination of transportation assistance and activities to raise public awareness, while another project indicated the consortium would be eliminated in the absence of sustained funding, a major setback given that it serves as the main vehicle for interagency collaboration.

\section{Key Community Themes}

\section{Cultural Competence}

While no two projects define cultural competence the same, they share an understanding that it is much broader than addressing the demographics of the people served in their communities. As one respondent noted, "It's an ongoing process to make sure we're addressing the needs of clients we are charged with serving. Every day there are unique situations that are related to cultural competence."

All eight projects noted that staff training is a key effort in addressing cultural competence. Five projects indicated they established relationships with individuals or cultural organizations that serve their target population such as traditional healers, faith-based organizations, and ethnic associations. Six projects included bilingual staff or had interpreters available. Similarly, five projects distributed written materials in two or more languages. One project described four cultural support groups they initiated-Vietnamese, Sudanese, Hispanic, and Englishspeaking. 
All eight projects felt they have been effective in demonstrating cultural competence, as demonstrated by their anecdotal accounts of increased enrollment, reduced no shows, and client retention. However, there is widespread recognition that more can be done. For example, projects indicated they need to target efforts to subpopulations including Latinas (three projects), homeless individuals (two projects), drug addicted women (two projects), and Hmong (one project). One project reported the challenge of reaching undocumented immigrant women and several projects acknowledged that language barriers remain an ongoing challenge despite gains they've made. Finally, projects noted that they are sometimes confronted with cultural practices that contradict best $\mathrm{MCH}$ practices. For example, one project indicated that they serve many participants whose cultural belief is that pregnant women should not be examined by a doctor. Racism, classism, and sexism were additional barriers noted by three projects.

\section{Community Involvement/Community Voice}

One of the three core goals of the Healthy Start program is the notion of community voice or community involvement, which refers to the input and incorporation of feedback from residents and service providers in Healthy Start's geographic service areas [9]. Projects identified this concept as critical to their success and many of them have developed mechanisms for understanding and engaging community voice, including focus groups with participants to assess the community's needs and why women seek care (four projects) and consumer involvement on the consortium (three projects). Community voice is also encouraged by holding town meetings and community forums (two projects), involving community members in local evaluation activities such as survey development, data collection, and presentations (two projects), having participants assist in the development of public awareness materials (one project), and employing former participants who are engaged in the community (one project).

Projects identified several benefits of obtaining and using the voice of participants and the larger community. Respondents noted that incorporating community voice was influential in increasing projects' understanding of the health care system and social challenges facing communities, advancing recommendations for change, and implementing solutions related to reducing infant mortality. Projects also indicated that consumer input at local consortium meetings contributes to the discussion of perinatal issues with State public health officials, providers, and project planners. The only challenge noted in this area was that of capturing the voice of homeless persons given their transient nature (two projects).
Table 1 Perceptions of components that contributed to achievement of goals or reducing disparities in birth outcomes ${ }^{3}$

\section{A}

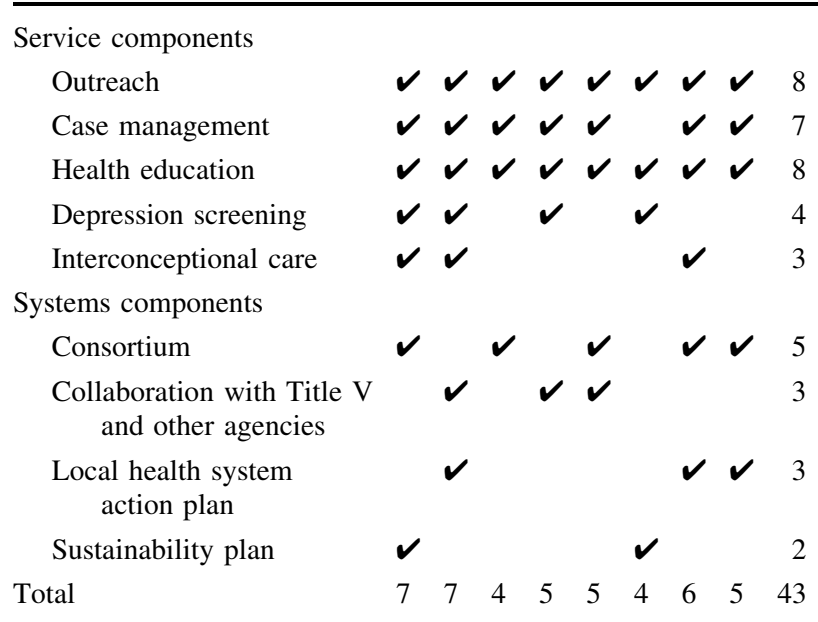

Grantees' Perceptions of Components that Contributed to Achievement of Goals or Reducing Disparities in Birth Outcomes

Table 1 presents respondents perceptions of components that contributed to achievement of their goals or reducing disparities in birth outcomes obtained during individual interviews and the project director mapping exercise. Outreach, case management, and health education are the required service components most commonly attributed to projects' self-perceived achievements. In fact, seven projects identified these three components as major contributing factors. Together, these services connect women with needed resources, often meeting their basic needs where there is great demand given the context in which the projects are operating. In addition, they connect women with social supports by establishing ongoing relationships that build trust and encourage their continued participation. They are key services for reaching and keeping women engaged. Depression screening and interconceptional care, the "youngest" required service components of the program, were cited less often as contributing factors.

Overall, systems components are less often reported as contributing factors to perceived achievements. Among them, consortium was the most noted systems component

\footnotetext{
${ }_{3}$ Categories are based on grouped and coded open-ended responses to "Which components contributed most to the achievement of your goals?" "Which specific components were most influential in the success of the activities that you have linked to reducing disparities in birth outcomes?" "Thinking about your prenatal and interconceptional services, what have been your most effective strategies to reduce disparities?" and "What have been the most effective strategies in the program to reduce disparities in infant mortality?"
} 
Table 2 Grantees perceived achievements ${ }^{4}$

\begin{tabular}{|c|c|c|c|c|c|c|c|c|c|}
\hline & A & $\mathrm{B}$ & $\mathrm{C}$ & $\mathrm{D}$ & $\mathrm{E}$ & $\mathrm{F}$ & G & $\mathrm{H}$ & Tota \\
\hline \multicolumn{10}{|l|}{ Service-related } \\
\hline $\begin{array}{l}\text { Increased participant } \\
\text { awareness }\end{array}$ & $\boldsymbol{V}$ & & & & $\checkmark$ & $\checkmark$ & & & 3 \\
\hline $\begin{array}{l}\text { Provision of enabling } \\
\text { services }\end{array}$ & & $\checkmark$ & & $\checkmark$ & & $\checkmark$ & $\checkmark$ & $\checkmark$ & 5 \\
\hline Universal screening & & & $\checkmark$ & & & & & $\checkmark$ & 2 \\
\hline Provision of male services & $\boldsymbol{V}$ & $\checkmark$ & & & & $\checkmark$ & & & 3 \\
\hline Increased service utilization & & $\boldsymbol{V}$ & & & $\checkmark$ & $\checkmark$ & & $\boldsymbol{V}$ & 4 \\
\hline $\begin{array}{l}\text { Increase in participants } \\
\text { w/medical home }\end{array}$ & $\checkmark$ & & & & & & $\boldsymbol{V}$ & & 2 \\
\hline $\begin{array}{l}\text { Earlier entry into prenatal } \\
\text { care }\end{array}$ & $\boldsymbol{V}$ & $\checkmark$ & $\boldsymbol{V}$ & $\checkmark$ & & $\checkmark$ & & & 5 \\
\hline \multicolumn{10}{|l|}{ Systems-related } \\
\hline $\begin{array}{l}\text { Increased provider/ } \\
\text { community awareness }\end{array}$ & $\boldsymbol{V}$ & $\checkmark$ & & & $\checkmark$ & $\checkmark$ & $\boldsymbol{V}$ & $\checkmark$ & 6 \\
\hline $\begin{array}{l}\text { Use of data for quality } \\
\text { improvement }\end{array}$ & $\checkmark$ & $\checkmark$ & & $\checkmark$ & & & $\checkmark$ & $\boldsymbol{V}$ & 5 \\
\hline Culturally diverse staff & $\boldsymbol{V}$ & $\checkmark$ & & $\checkmark$ & $\checkmark$ & $\checkmark$ & & $\checkmark$ & 6 \\
\hline Consumer involvement & $\boldsymbol{V}$ & $\checkmark$ & $\boldsymbol{V}$ & $\checkmark$ & $\checkmark$ & & & $\checkmark$ & 6 \\
\hline $\begin{array}{l}\text { Coordinated systems/ } \\
\text { services }\end{array}$ & $\boldsymbol{V}$ & $\checkmark$ & & $\checkmark$ & & $\checkmark$ & $\checkmark$ & $\checkmark$ & 6 \\
\hline Integrated service system & & $\checkmark$ & $\boldsymbol{V}$ & $\boldsymbol{V}$ & $\checkmark$ & & & $\checkmark$ & 5 \\
\hline \multicolumn{10}{|l|}{ Population change } \\
\hline Improved birth outcomes & $\checkmark$ & $\checkmark$ & $\boldsymbol{V}$ & $\checkmark$ & & & $\checkmark$ & $\checkmark$ & 6 \\
\hline Total & 10 & 11 & 5 & 8 & 6 & 8 & 6 & 10 & 64 \\
\hline
\end{tabular}

(five projects). The other systems components were less frequently listed-collaboration with Title $\mathrm{V}$ and other agencies (three projects), LHSAP (three projects) and sustainability planning (two projects).

\section{Grantees Perceived Achievements}

Table 2 shows the successes projects reported having achieved, from the perspective of various stakeholders. Projects cited both service and system level achievements, noting the latter with greater frequency. The provision of enabling services such as transportation and child care, and getting women into prenatal care earlier in pregnancy were the most frequently reported service-related achievements. The achievement of male services refers to care management or support groups offered to males in the community. Among systems-related achievements, increased provider community awareness, culturally diverse staff, consumer involvement, and coordinated systems and services were reported most frequently. Respondents indicated their projects evaluate data on a host of variables such as

\footnotetext{
$\overline{4}$ Categories are based on grouped and coded open-ended responses to "What have been [project's] most important achievements?" asked on multiple discussion guides.
}

Table 3 Challenges to project success cited by grantees ${ }^{5}$

\begin{tabular}{|c|c|c|c|c|c|c|c|c|c|}
\hline & A & $\mathrm{B}$ & $\mathrm{C}$ & $\mathrm{D}$ & $\mathrm{E}$ & $\mathrm{F}$ & $\mathrm{G}$ & $\mathrm{H}$ & Tot: \\
\hline \multicolumn{10}{|l|}{ Systems-level } \\
\hline Service availability & $\checkmark$ & & $\checkmark$ & $\checkmark$ & $\checkmark$ & & & $\checkmark$ & 5 \\
\hline Transportation & & & $\checkmark$ & & $\checkmark$ & & $\checkmark$ & & 3 \\
\hline $\begin{array}{l}\text { Complicated Medicaid } \\
\text { enrollment }\end{array}$ & & & & & $\checkmark$ & & & $\checkmark$ & 2 \\
\hline Culturally competent care & & & & $\checkmark$ & $\checkmark$ & $\checkmark$ & & $\checkmark$ & 4 \\
\hline \multicolumn{10}{|l|}{ Social context } \\
\hline Persistent/increasing poverty & & & $\checkmark$ & & & & $\boldsymbol{\nu}$ & & 2 \\
\hline Mobile population & & & $\checkmark$ & $\checkmark$ & & & $\checkmark$ & $\checkmark$ & 4 \\
\hline Domestic violence & & & $\checkmark$ & & & $\checkmark$ & & & 2 \\
\hline Funding challenges & $\boldsymbol{V}$ & $\checkmark$ & $\checkmark$ & & & $\checkmark$ & $\checkmark$ & & 5 \\
\hline Staff capacity & $\boldsymbol{V}$ & & $\checkmark$ & & & $\checkmark$ & & $\checkmark$ & 4 \\
\hline Consumer involvement & & & $\checkmark$ & & & & $\checkmark$ & $\checkmark$ & 3 \\
\hline Total & 3 & 1 & 8 & 3 & 4 & 4 & 5 & 6 & 34 \\
\hline
\end{tabular}

demographics, screening results, health behaviors, and health outcomes. Data are used to plan community events, establish benchmarks, target subpopulations, focus on areas for improvement, and measure effectiveness of services (e.g. health educations' effect on behaviors). Of note, improved birth outcomes (e.g. a reduction in infant mortality), a long term population outcome on the logic model, was self-reported as frequently as the most commonly reported intermediate outcomes for project participants.

\section{Grantees' Noted Challenges}

Projects reported a wide range of both contextual and organizational challenges to achieving program outcomes. The number of self-reported challenges varied considerably across grantees, from one to eight. Service availability (or lack thereof) and funding were the most frequently reported challenges (Table 3). Staff capacity, reported by half of the projects, refers to the large caseloads maintained by case managers/care coordinators and the subsequent implications on the frequency and duration of their contacts with participants. This is also linked to the funding challenges faced by the projects, which prevents them from hiring more staff to relieve caseload burden.

Consumer/community involvement was a challenge noted by three projects. Interestingly, it was also identified as an achievement (Table 2) noted by the majority of projects, suggesting it is an area in which projects have

\footnotetext{
${ }^{5}$ Categories are based on grouped and coded open-ended responses to "What have been [project's] organizational challenges to serving women during the prenatal period" and "What external contextual factors have influenced the success of the [project]'s initiative in your community?" asked on multiple discussion guides.
} 
made notable strides, but is an area still requiring improvement.

\section{Discussion}

Reducing infant mortality and racial disparities are major challenges that require multiple strategies within a community context. It is especially challenging in communities with limited resources, resulting in services' and systems' gaps for high risk women and their families. In-depth site visits with eight Healthy Start grantees provided an opportunity to learn how grantees organize services and promote systems of care. Although the eight selected sites implement the nine required components, there is variability in the perceived contributions of the components to project achievements. Although the ultimate goals of each project may be similar, methods to achieve them and emphases are different. The unique cultural and community context of each project must not be overlooked as they influence projects' design, implementation and successes.

Findings from the site visits reveal that projects report service components more often as contributing to achievements. Interestingly, however, these supported more self-reported systems-related achievements (e.g. increased collaboration, improved cultural competence) than services-related achievements (e.g. number of referrals made; frequency of prenatal visits, etc.). As one of the first national programs to emphasize systems development, these findings support the importance of Healthy Start project work to develop integrated service systems and community voice such that sustainable community-based systems of care are rooted and implemented in the community. In addition to reporting several intermediate outcomes such as increased service utilization and improved coordination and collaboration, projects also reported longer term outcomes such as an integrated service system and improved birth outcomes. These reported findings, however, were not verified by examining local evaluation data.

Making associations about which components lead to different outcomes is challenging given that the components do not operate independently. For example, case managers and outreach workers conduct health education; outreach workers provide case management services. The staffing design is such that individuals wear many hats and their staff roles are interconnected.

Among systems components, respondents identified consortia as the most central to projects' reported achievements. Healthy Start has had an emphasis on establishing and maintaining community consortia since it began in 1991 [10]. Site visits underscored that the most common method for collaboration with Title $\mathrm{V}$ and other entities is via inclusion on the local consortium, signifying that these two systems activities may be not be as separate as the current Healthy Start program design implies. Sustainability planning was not a priority area for most projects.

While projects have made progress in designing and implementing their services and systems with noted success, they continue to be faced with challenges. Limited funding is often cited. "We have to do more with less" was echoed by several respondents. Also challenging are the social and systems-oriented barriers such as the mobility of the target population, limited staff capacity such as mental health service providers, and transportation barriers. These challenges, consistently identified during the site visits, do not have easy fixes. They require consistent support for social policies that meet the basic needs for participants. They also depend on communities having an infrastructure in place (e.g. sufficient subsidized housing; public transportation) that allows for the provision of such necessities.

Findings from these site visits support the links between the program components and intermediate program outcomes hypothesized in the logic model at the beginning of the evaluation, as well as project directors' perceptions of long term outcomes. Results emphasized the importance of both service and system components and the project-specific ways of implementing the components in each community. Unlike previous Healthy Start evaluations, this one included a focus on systems components of community-based programs. These findings support systems development as a key factor in influencing project success.

A limitation of using a case study methodology is that by design, it cannot point to causal relationships between program components and long term outcomes. Another limitation of the study is that accomplishments reported by project directors were not verified through the examination of local evaluation data given that data reporting is inconsistent and incomplete.

Site visits provided insight about eight selected grantees; findings cannot be generalized to the entire Healthy Start grantee population. Since the 96 projects vary across a host of characteristics including project design, population demographics, collaborative relationships, and available resources, findings from the site visits serve as a reminder of the different contexts and community issues faced by each project. Although the sample is small and not representative of all Healthy Start projects, it is encouraging that the eight projects are reporting improved outcomes among the participants they serve.

The eight projects provided insight into how local sites really use a national program. We saw a great deal of effort at the local level that would not have been appreciated without going on-site and speaking with several stakeholders to understand the nuts and bolts of project nuances. This study is but one piece of a larger endeavor to 
understand the effects of the Healthy Start program. Future evaluations of Healthy Start should continue to examine the ways communities implement the service and systems components and their relationship to long-term goals for sustainable systems and improved health outcomes for mothers and infants.

Acknowledgments This research was supported by the Maternal and Child Health Bureau, Health Resources and Services Administration under Contract No. 233-02-0088 Task Order \#11. The authors are grateful for the ongoing support from David de la Cruz and the contributions made by the Healthy Start Panel. A special note of thanks to the staff and volunteers at the eight Healthy Start projects who participated in the site visits.

\section{References}

1. Mathews, T. J., \& MacDorman, M. F. (2007). Infant mortality statistics from the 2004 period linked birth/infant death data set. National Vital Statistics Reports, 55(14), 1-31.

2. Health Resources and Services Administration. (2008). A profile of healthy start: Findings from phase I of the evaluation. Rockville: HRSA, Maternal and Child Health Bureau.
3. Health Resources and Services Administration. (2001). Application guidance for the following four healthy start initiative competitions. Rockville: HRSA, Maternal and Child Health Bureau.

4. Kish, L. (1987). Statistical design for research. New York: Wiley.

5. Miles, M. B., \& Huberman, A. M. (1994). Qualitative data analysis thousand oakes. Thousand oaks, CA: Sage Publications.

6. Betancourt, J. R., et al. (2005). Cultural competence and health care disparities: Key perspectives and trends. Health Affairs, 24(2), 499-505. doi:10.1377/hlthaff.24.2.499.

7. Howell, E. M., et al. (1998). Back to the future: Community involvement in the Healthy Start program. Journal of Health Politics, Policy and Law, 23(2), 291-317.

8. Elliot, S. A., et al. (2001). Training health visitors to reduce levels of depression after childbirth: An evaluation. Journal of Mental Health, 10(6), 613-625. doi:10.1080/09638230120041362.

9. Plough, A., \& Olafson, F. (1994). Implementing the Boston Healthy Start Initiative: A case study of community empowerment and public health. Health Education Quarterly, 21(2), 221-234.

10. McCoy-Thompson, M. (1994). The healthy start initiative: A community-driven approach to infant mortality reduction-vol. I Consortia development. Arlington: National Center for Education in Maternal and Child Health. 